\title{
Associative learning shapes visual discrimination - a web-based study
}

\author{
Yannik Stegmann1, Marta Andreatta1,2, Paul Pauli1,3, Matthias J. Wieser² \\ ${ }^{1}$ Department of Psychology (Biological Psychology, Clinical Psychology, and Psychother- \\ apy), University of Würzburg, Germany \\ 2 Department of Psychology, Education, and Child Studies, Erasmus University Rotter- \\ dam, Netherlands \\ ${ }^{3}$ Center for Mental Health, Medical Faculty, University of Würzburg, Germany
}

\begin{abstract}
Threat detection plays a vital role in adapting behavior to changing environments. A fundamental function to improve threat detection is by learning to differentiate between stimuli predicting danger and safety. Accordingly, aversive learning should lead to enhanced sensory discrimination of danger and safety cues. However, studies investigating the psychophysics of visual and auditory perception after aversive learning show divergent findings, and both enhanced and impaired discrimination after aversive learning have been reported. Therefore, the aim of this web-based study is to examine the impact of aversive learning on a continuous measure of visual discrimination. To this end, $\mathrm{n}=205$ participants underwent a differential fear conditioning paradigm before and after completing a visual discrimination task using differently oriented grating stimuli. Participants saw either unpleasant or neutral pictures as unconditioned stimuli (US). Results demonstrated sharpened visual discrimination for the US-associated stimulus (CS+), but not for the unpaired conditioned stimuli (CS-). Importantly, this finding was irrespective of the US's valence. These findings suggest that associative learning results in increased stimulus salience, which facilitates perceptual discrimination in order to prioritize attentional deployment.
\end{abstract}

\section{Introduction}

Learning to detect threat-predicting stimuli is a fundamental function for supporting adaptive behavior in ever-changing environments. Recent theories propose that threat learning prompts changes in sensory processing to improve discrimination 
between threat- and safety-related signals. These changes can manifest on every stage of the sensory system hierarchy and have already been documented for a wide range of modalities. For example, Kass et al. (2013) observed facilitated synaptic output of olfactory sensory neurons in rodents after selectively pairing odors with electrical stimuli. Similar results could be demonstrated in humans by using initially indistinguishable odor cues in an aversive learning paradigm. Functional imaging analyses showed that olfactory threat learning modified neural organization in the primary olfactory cortex, which was accompanied by enhanced behavioral discrimination of threat-related odors (Li et al., 2008). Following threat learning, auditory cortex neurons showed enhanced responsiveness to threat-predicting tones in rats (Quirk et al., 1997), rabbits (Kraus \& Disterhoft, 1982), and cats (Diamond \& Weinberger, 1986). These findings are paralleled by results of studies measuring neural activity of the human auditory cortex during threat learning by the means of functional magnetic resonance imaging (fMRI; Apergis-Schoute et al., 2014), magnetoencephalography (MEG; Kluge et al., 2011), and electroencephalography (EEG; Heim \& Keil, 2006), demonstrating unequivocal evidence for increased response amplitudes to threat-predicting stimuli.

Studies investigating the visual sensory system yield comparable results (see Miskovic \& Keil, 2012 for a review). Using EEG as a direct measure of visuocortical activity, numerous studies demonstrated converging evidence for enhanced sensory processing of threat-related features of visual stimuli. Mirroring the organization of visual receptive fields, threat-related sensory amplification has been reported for visual stimuli differing in orientation (McTeague et al., 2015; Miskovic \& Keil, 2013), spatial frequency (Friedl \& Keil, 2019), location (Friedl \& Keil, 2020), and color (Boylan et al., 2019). However, enhanced visuocortical responses have also been observed for more complex visual stimuli, like geometric symbols (Stegmann et al., 2019; Wieser et al., 2016), pictures of virtual rooms (Kastner et al., 2015) and facial identities (Stegmann et al., 2020; Wieser et al., 2014).

Together, these studies support the notion that sensory systems are dynamically adapting in response to environmental cues signaling motivational significance (Lang et al., 1997). However, the extent to which these adaptions in the sensory cortices are accompanied by changes in behavioral performance remains elusive and recent studies investigating the psychophysics of visual and auditive discrimination during aversive learning showed mixed results. Contrary to expectations, Resnik et al. (2011) found an increase 
in discrimination thresholds using auditive stimuli paired with aversive odors or sounds. The authors suggest that aversive learning prompts generalization processes in sensory systems to widen responses to upcoming danger, thus lowering visual discrimination. In a similar line of studies, the authors could retrieve this pattern for visual stimuli differing in orientation or contrast followed by aversive picture stimuli (Shalev et al., 2018). On the other hand, Rhodes et al. (2018) showed decreased discrimination thresholds using differently oriented grating stimuli followed by aversive noise blasts. Importantly, this improved visual discrimination was specific for the grating stimulus paired with the aversive sound and was not found for other orientations.

Despite their methodological similarities these studies show divergent results. Both lines of studies employed a differential fear conditioning paradigm and measured the change of discrimination acuity using a psychophysical staircase approach to identify the just noticeable difference threshold (JND). Even though the JND is a well-established psychophysical index (see also Holt et al., 2014), it may have a limited use in capturing sensory changes in aversive learning paradigms. First, the JND is only a point measure of discrimination acuity, thus obscuring potential nongradual effects on visual discrimination along the perceptual continuum. These nongradual effects have already been identified, for example, for visuocortical activation patterns during aversive generalization learning paradigms using grating stimuli (Antov et al., 2020; McTeague et al., 2015) or faces (Stegmann et al., 2020). Second, the JND procedure varies in the number of unreinforced presentations of the conditioned stimulus, depending on the participant's response pattern. This might be problematic as longer procedures are more affected by the temporal dynamics of extinction learning. This is aggravated by the observation that threatrelated visuocortical sharpening extinguishes rapidly (McTeague et al., 2015).

To overcome these issues, we used a yes-no task to measure visual discrimination along a continuum of radial distance in steps of $1^{\circ}$ before and after differential aversive learning. The goals of this study were threefold: (1) Based on recent demonstrations delivering fear conditioning tasks remotely (McGregor et al., 2021; Purves et al., 2019), we wanted to establish a web-based discrimination and aversive learning task, exploiting the benefits of quickly and economically collecting large samples outside of the laboratory context. (2) Given the mixed results regarding how aversive learning influences discrimination acuity, we tested the hypothesis that aversive learning changes visual discrimination for the CS+, but not the CS- orientation. (3) To further test the hypothesis that 
discriminative learning improves discrimination accuracy, we included two further groups that did the same discrimination task as the differential learning groups but saw six additional generalization stimuli (GS) in $10^{\circ}$ steps around the CS+ $\left(-30^{\circ},-20^{\circ},-10^{\circ}, 10^{\circ}\right.$, $20^{\circ}, 30^{\circ}$; see also McTeague et al., 2015) during aversive learning. We expected that these groups exhibit even higher discrimination accuracies after learning, since they experience that only the CS+ is followed by an US, while all other GS, even those perceptually similar to the CS+, signal safety.

\section{Methods}

\section{Preregistration and online data}

The hypotheses and methods of this study were preregistered. The preregistration, all data and analyses are publicly available at https://osf.io/4cz2e.

\section{Participants}

From a total of 336 individuals 205 (65,2\%) subjects (169 females) with a mean age of $23.8 \pm 7.1$ years completed the web-based study. Participants were required to be older than 18 years and be free of a lifetime history of mental disorders (as assessed by self-report). After completing demographic and anxiety questionnaires, participants were randomly allocated to one of six experimental groups. There were four groups that completed a standard differential fear conditioning paradigm (Diff), while two additional groups underwent a modified fear generalization paradigm (Gen). Two of the differential learning groups were tested on the unpaired conditioned stimulus (control group, c) in the discrimination task. Groups were either presented with aversive (+) or neutral (-) US. Characteristics for the six resulting groups are summarized in Table 1. All experimental procedures were approved by the ethics committee of the Department of Psychology at the University of Würzburg. All participants provided informed consent online. They received either course credits or could join a lottery for one out of five $15 €$ coupons as compensation. 
Table 1: Summary of group characteristics.

\begin{tabular}{|c|c|c|c|c|c|c|c|c|}
\hline & & & Gro & ups & & & & \\
\hline & Diff+ & Diff- & Gen+ & Gen- & cDiff+ & cDiff- & $F(5,199)$ & $\mathrm{p}$ \\
\hline $\mathrm{n}$ & 31 & 37 & 37 & 36 & 32 & 32 & & \\
\hline Women (\%) & 77.4 & 94.6 & 70.3 & 86 & 81.2 & 84.4 & 1.73 & .129 \\
\hline Age & $\begin{array}{l}23.7 \\
(6.1)\end{array}$ & $\begin{array}{c}26.0 \\
(10.3)\end{array}$ & $\begin{array}{l}25.4 \\
(8.7)\end{array}$ & $\begin{array}{l}23.9 \\
(4.7)\end{array}$ & $\begin{array}{l}22.4 \\
(5.8)\end{array}$ & $\begin{array}{l}20.6 \\
(2.7)\end{array}$ & 2.71 & .021 \\
\hline BAI & $\begin{array}{c}11.3 \\
(10.1)\end{array}$ & $\begin{array}{c}12.3 \\
(9.4)\end{array}$ & $\begin{array}{l}13.2 \\
(9.9)\end{array}$ & $\begin{array}{c}11.5 \\
(11.0)\end{array}$ & $\begin{array}{c}15.4 \\
(13.3)\end{array}$ & $\begin{array}{c}13.5 \\
(10.5)\end{array}$ & .64 & .669 \\
\hline UI & $\begin{array}{c}48.1 \\
(14.6)\end{array}$ & $\begin{array}{c}46.5 \\
(12.6)\end{array}$ & $\begin{array}{c}49.1 \\
(15.8)\end{array}$ & $\begin{array}{c}45.8 \\
(15.6)\end{array}$ & $\begin{array}{c}49.8 \\
(13.1) \\
\end{array}$ & $\begin{array}{c}48.8 \\
(11.1)\end{array}$ & .43 & .827 \\
\hline US arousal & $\begin{array}{c}70.1 \\
(16.0)\end{array}$ & $\begin{array}{c}26.5 \\
(20.7)\end{array}$ & $\begin{array}{c}70.4 \\
(18.5)\end{array}$ & $\begin{array}{c}23.3 \\
(17.9)\end{array}$ & $\begin{array}{c}69.7 \\
(24.4)\end{array}$ & $\begin{array}{c}28.5 \\
(21.0)\end{array}$ & 50.5 & $<.001$ \\
\hline US unpleasantness & $\begin{array}{c}71.4 \\
(21.1) \\
\end{array}$ & $\begin{array}{r}49.4 \\
(8.8) \\
\end{array}$ & $\begin{array}{c}75.7 \\
(15.1) \\
\end{array}$ & $\begin{array}{r}47.9 \\
(9.3) \\
\end{array}$ & $\begin{array}{c}74.4 \\
(15.2) \\
\end{array}$ & $\begin{array}{c}44.4 \\
(14.9)\end{array}$ & 35.6 & $<.001$ \\
\hline Memory scores & $\begin{array}{c}8.5 \\
(3.3)\end{array}$ & $\begin{array}{c}9.7 \\
(1.5)\end{array}$ & $\begin{array}{c}8.5 \\
(3.0)\end{array}$ & $\begin{array}{c}9.1 \\
(2.0)\end{array}$ & $\begin{array}{l}9.25 \\
(1.9)\end{array}$ & $\begin{array}{l}8.56 \\
(3.1)\end{array}$ & 1.41 & .224 \\
\hline
\end{tabular}

Diff = differential learning group, Gen = generalization learning group, $\mathrm{c}=$ control discrimination task (CS-) + + = aversive US, - = neutral US, BAI = Becks Anxiety Inventory, UI = Uncertainty Intolerance. Numbers indicate means ( \pm s.d.).

\section{Materials}

Circular black-and-white sinusoidal grating stimuli $(10 \mathrm{~Hz}$ spatial frequency) filtered with a Gaussian-envelope (i.e., Gabor-patch) with maximum contrast of $100 \%$ at center were used as conditioned stimuli for the conditioning procedure and as target stimuli for the visual discrimination task. Aversive and neutral picture stimuli served as unconditioned stimuli (US). All pictures were extracted from the OASIS data set (Kurdi et al., 2017). Ten high arousing/unpleasant (I26, I209, I208, I714, I276, I855, I210, I496, I287, I440) and ten low arousing/neutral (I182, I597, I602, I673, I181, I891, I596, I195, I632, I594) pictures were chosen according to their normative ratings for arousal and valence. To ensure that participants perceived the aversive or neutral picture stimuli as such, they rated the picture stimuli for subjective arousal ("As how arousing do you perceive this picture?", $0=$ low arousing, calm to 100 = very arousing) and valence ("As how pleasant/unpleasant do you perceive this picture?", $0=$ very pleasant, $100=$ very 
unpleasant) using visual analogue scales at the end of the experimental session. All stimuli were presented centrally on a grey background $(\mathrm{RGB}=128,128,128)$. Please note, that we could not control the participants' monitor setup in this web-based study. However, the experiment did not run on tablets or mobile devices.

\section{Design}

After giving informed consent, participants completed German versions of a demographic questionnaire, Becks-Anxiety-Inventory (BAI; Beck et al., 1988) and Uncertainty Intolerance questionnaire (UI-18; Buhr \& Dugas, 2002; Gerlach et al., 2008) , using an online survey platform (www.soscisurvey.de). They were then redirected to www.pavlovia.org, where the main experiment took place (see Fig. 1A). The discrimination task consisted of 160 trials, each starting with a 1,500 ms - 2,500 ms presentation of a centrally presented fixation cross, before the first Gabor-patch was presented for 500 ms (see Fig. 1B). The Gabor-patch was masked by a scrambled stimulus (200 ms) before the second Gabor-patch was presented for 500 ms. Either the first or the second Gabor-patch was always the target stimulus (CS+ in the experimental groups, CS- in the control groups). Beginning with the onset of the second Gabor-patch, participants had up to 3,000 ms for responding weather the Gabor-patches were identical (Press ' $\mathrm{X}$ ') or not (Press ' $\mathrm{N}$ '). The orientation of the Gabor-patches varied between $-30^{\circ}$ to $30^{\circ}$ in steps of $1^{\circ}$ around the target stimulus. Since we assumed that effects of aversive learning on visual discrimination would be most likely evident in orientations perceptually similar to the target orientation, the number of presentations per orientation followed a normal distribution around the target orientation (i.e., more presentations of orientations close to the target orientation, see also Fig. 2A). Participants did not receive feedback except for 20 practice trials at the beginning of the experiment.

Next, all participants completed an aversive learning paradigm according to their group condition. The differential learning groups (Diff+, Diff-, cDiff+ and cDiff-) were presented with 40 trials of CS+ and 40 trials of CS- (80 trials in total). The generalization learning groups (Gen+, Gen-) were presented with 10 trials of each CS+, CS- and six generalization stimuli (GS) in $10^{\circ}$ steps around the $C S+\left(-30^{\circ},-20^{\circ},-10^{\circ}, 10^{\circ}, 20^{\circ}, 30^{\circ}\right.$; see also McTeague et al., 2015), resulting in 80 total trials. In both versions, each CS+ presentation was associated with an US (100\% reinforcement rate), while the CS- and GS remained 
unpaired. Importantly, five of the ten aversive picture stimuli served as US for the aversive learning groups (Diff + , Gen+, cDiff + ), whereas five of the ten neutral picture stimuli were used as US for the neutral learning groups (Diff-, Gen-, cDiff-). The remaining five picture stimuli of the same category were used in the US memory task at the end of the experiment. The choice of US was counter-balanced between participants. During each trial, the CS was presented for 2,000 ms and was followed by a 1,000 ms presentation of the US in case of the CS+. The duration of the ITI varied between 2,000 ms and 3,000 ms (see Fig. 1C). Participants were not instructed about the CS-US contingencies.

Before and after associative learning, every participant rated the CS and all GS for subjective threat ('How threatening do you perceive this stimulus?') on a visual analogue scale ranging from $0=$ 'not threatening at all' to $100=$ 'very threatening'. US-expectancy ratings ('How much do you expect the presentation of a picture after this stimulus?'; $0=$ 'not at all'; 100 = 'very likely') were collected after associative learning only.

Afterwards, participants repeated the visual discrimination task. At the end of the experiment, participants were submitted to a US-validation task, consisting of the US valence- and arousal-rating and a memory task. During the memory task, participants were confronted with the five picture stimuli, which served as US, in addition to the five remaining, novel stimuli. For each stimulus, participants had to answer (yes/no) if they had encountered this picture during the experimental session. Each correct answer was summarized, resulting in a memory score of zero to ten correctly remembered stimuli. 


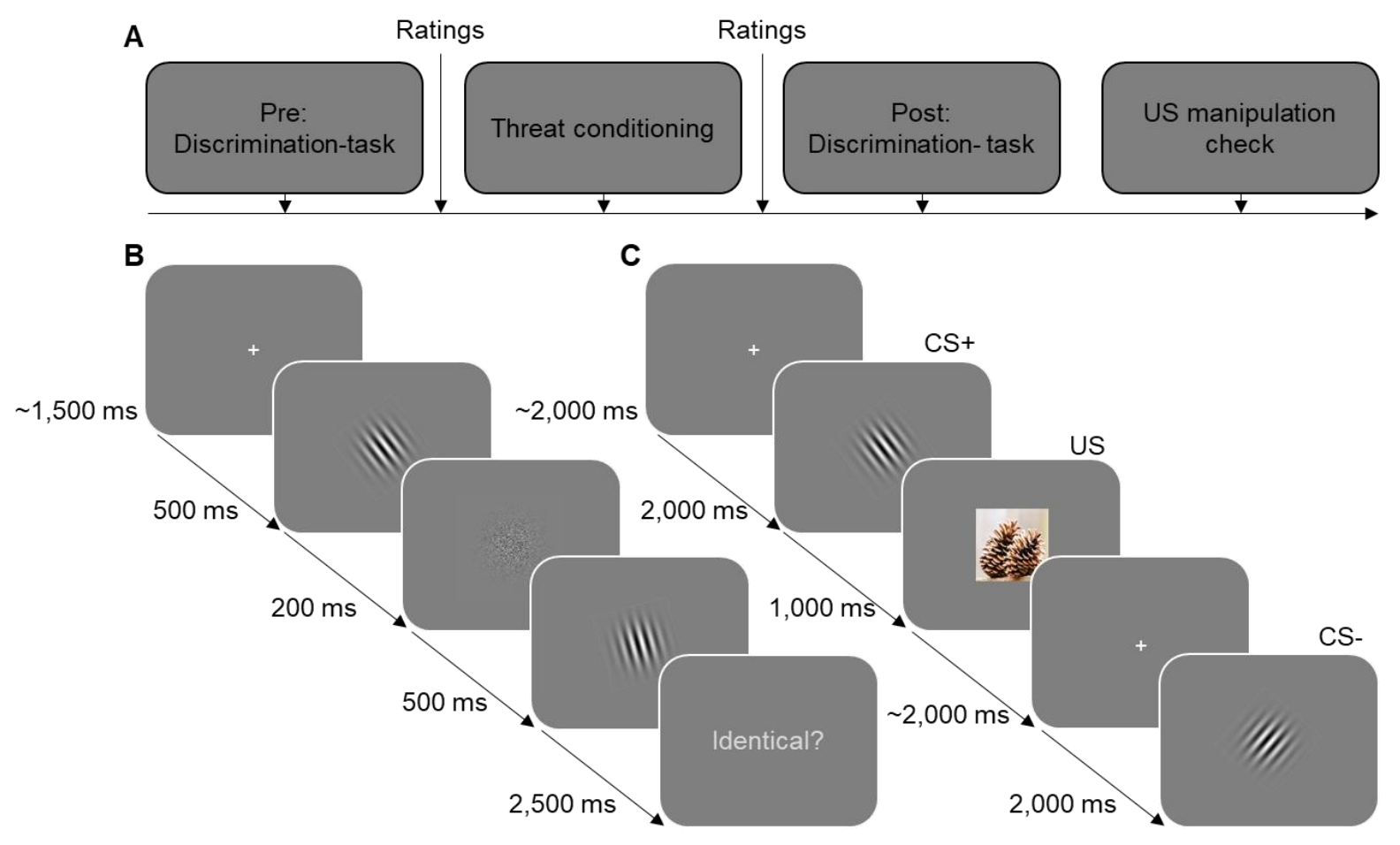

Figure 1: A) Flowchart of the general experimental design. B) Trial structure and timing of the visual discrimination task. C) Trial structure and timing of the associative learning task.

\section{Discrimination task and data analysis}

For behavioral analysis, all 'identical'-responses were collected and summarized per condition, phase and individuum (see Fig. 2A). To obtain a single measure of discrimination acuity for each phase and individuum, unimodal distributions were fitted to the individual response data (see Fig. 2B). Then, individual means and standard deviations of the Cauchy-distributions were extracted, using the 'fitdist'-algorithm of the 'fitdistrplus'package (Delignette-Muller \& Dutang, 2015) in R (version 4.3.0; R Development Core Team). Cauchy-distributions were used instead of standard-normal distributions, as they are more robust to outliers for conditions at the ends of the continuum, which were presented only once (Johnson et al., 1995). Importantly, the width of a Cauchy-distribution is represented by its standard deviation. Thus, smaller standard deviations indicate better visual discrimination. Therefore, the standard deviations of these distributions serve as an index of discrimination ability and the change of standard deviations can be used to measure the impact of associative learning on discrimination acuity. 

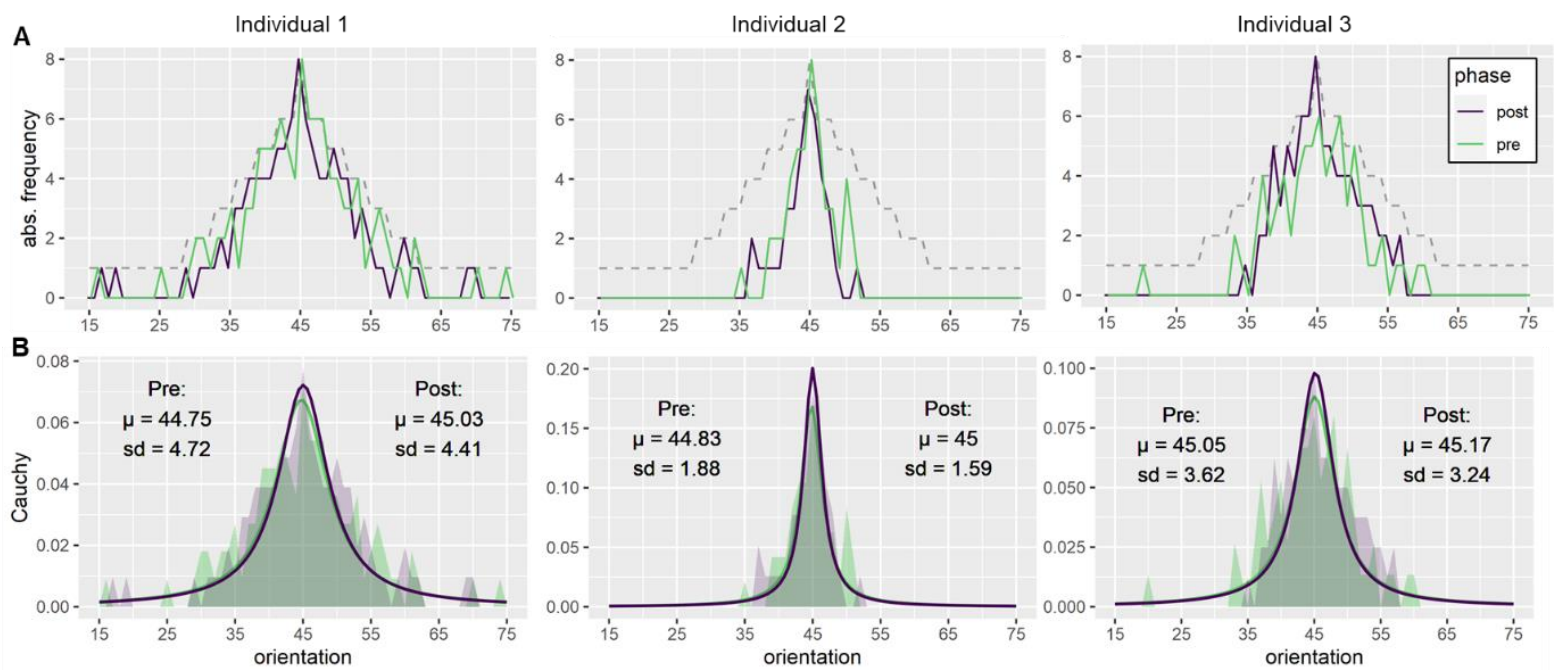

Figure 2: A) Three examples of individual response data from the visual discrimination task. The green and purple lines represent the sum of 'identical'-responses per condition during the pre- and post-task, respectively. The dotted line depicts the number of total presentations per condition. B) Bold lines show the fitted Cauchy-distributions over the behavioral response data (shaded areas) prior and after associative learning. The extracted means $(\mu)$ and standard deviations (sd) are noted in the diagram.

\section{Statistical analysis}

Aggregated data from the discrimination task were submitted to two separate lines of analyses. (1) To compare the different learning paradigms, we conducted a 2 (learning type: differential vs generalization learning) x 2 (aversiveness: neutral vs aversive US) $\mathrm{x}$ 2 (phase: pre vs post) ANOVA on the individual standard deviations. For this analysis, we included data from the Diff+, Diff-, Gen+ and Gen- groups. (2) To investigate the effect of US-contingency on visual discrimination, we run a 2 (target: CS+ vs CS-) x 2 (aversiveness: neutral vs aversive) $\times 2$ (phase: pre vs post) ANOVA. Here, we included data from the Diff+, Diff-, cDiff+ and cDiff- groups.

Similarly, for analyses of US-expectancy and subjective threat ratings, we conducted (1) 2 (learning type: differential vs generalization learning) x 2 (aversiveness: neutral vs aversive US) x 8 (orientation: $-30^{\circ},-20^{\circ},-10^{\circ}, \mathrm{CS}+,+10^{\circ},+20^{\circ},+30^{\circ}, \mathrm{CS}-$ ) ANOVAs and (2) 2 (target: CS+ vs CS-) x 2 (aversiveness: neutral vs aversive US) x 8 (orientation: $-30^{\circ},-20^{\circ},-10^{\circ}, \mathrm{CS}+,+10^{\circ},+20^{\circ},+30^{\circ}, \mathrm{CS}-$ ) ANOVAs. Please note that for rating analyses, 
the factor phase was omitted since US-expectancy ratings were only collected after associative learning.

Significant effects were followed up with $t$-tests. For rating analyses, post-hoc tests for the factor orientation were referenced against the CS- as is frequently done in the fear conditioning literature (Andreatta et al., 2020; Lissek et al., 2010). A significance level of 0.05 was used for all analyses and Greenhouse-Geisser correction was applied where appropriate (Greenhouse \& Geisser, 1959). Throughout this manuscript, corrected degrees of freedom, corrected $p$ values and the partial $\eta^{2}\left(\eta_{p}^{2}\right)$ or Cohen's $d(d)$ and their 95\% confidence interval are reported (Picton et al., 2000).

\section{Results}

\section{Discrimination task}

The 2 (learning type: differential vs generalization learning) x 2 (aversiveness: neutral vs aversive US) x 2 (phase: pre vs post) ANOVA of the standard deviations extracted from the discrimination task yielded a main effect of phase, $F(1,137)=8.13, p=$ $.005, \eta_{p}^{2}=.06$ [CI: 0.01; 0.14], and a marginal main effect of learning type, $F(1,137)=3.01$, $p=.085, \eta_{p}^{2}=.02$ [CI: 0.00; 0.09], which were further qualified by an interaction effect between phase and learning type, $F(1,137)=15.14, p<.001, \eta_{p}^{2}=.10$ [CI: 0.02; 0.20]. Other effects were not significant, $p s>$.357. Post-hoc $t$-tests revealed a decrease in standard deviations, and therefore, improved discrimination acuity after associative learning for the groups Diff $+, t(30)=-3.44, p=.002, d=-0.62$ [CI: $-1.00 ;-0.23$ ], and Diff-, $t(36)=-$ $4.74, p<.001, d=-0.78$ [CI: $-1.14 ;-0.41]$, but not for Gen $+, t(36)=0.20, p=.841, d=0.03$ [CI: $-0.29 ; 0.36$ ], and Gen-, $t(35)=0.79, p=.437, d=0.13$ [CI: $-0.20 ; 0.46$ ]. These results indicate sharpened visual discrimination after associative learning in the differential learning groups, but not in the generalization learning groups, independent of the US valence (see Fig. 3 and 4).

To test the specificity of this effect, the differential learning groups were compared to the control groups, who underwent the same learning paradigm but were psychophysically tested on the CS-. The 2 (target: CS+ vs CS-) x 2 (aversiveness: neutral vs aversive US) x 2 (phase: pre vs post) ANOVA revealed a main effect of phase, $F(1,128)=11.42, p<$ $.001, \eta_{p}^{2}=.08$ [CI: 0.01; 0.18], which was again further qualified by a significant 
interaction between target and phase, $F(1,128)=13.99, p<.001, \eta_{p}^{2}=.10$ [CI: $\left.0.02 ; 0.20\right]$. No other effect was significant, $p s>.465$. The control groups showed no changes in discrimination acuity due to associative learning, cDiff+: $t(31)=-0.04, p=.971, d<0.01$ [CI: $-0.35 ; 0.34$ ], cDiff-: $t(31)=0.41, p=.688, d=0.07$ [CI: $-0.28 ; 0.42$ ].
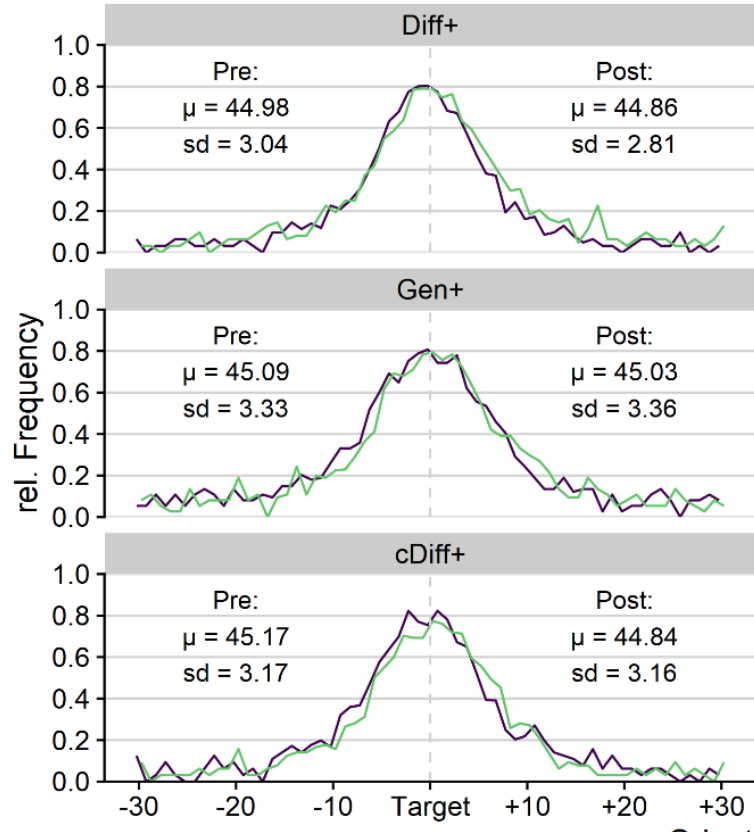

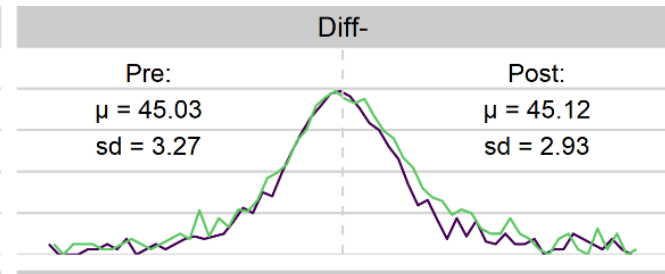

Gen-

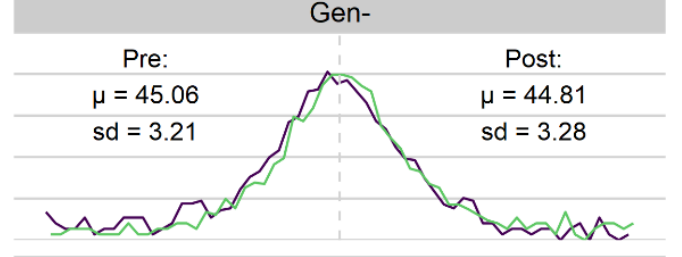

Phase

- post

Figure 3: Group-averaged response distributions during the discrimination tasks. The proportion of 'identical'-responses per orientation were calculated as relative frequency. The dotted vertical line represents the target orientation (CS+ for Diff and Gen, CS- for cDiff). The grand averages of the extracted means $(\mu)$ and standard deviations (sd) are noted in the diagram. 


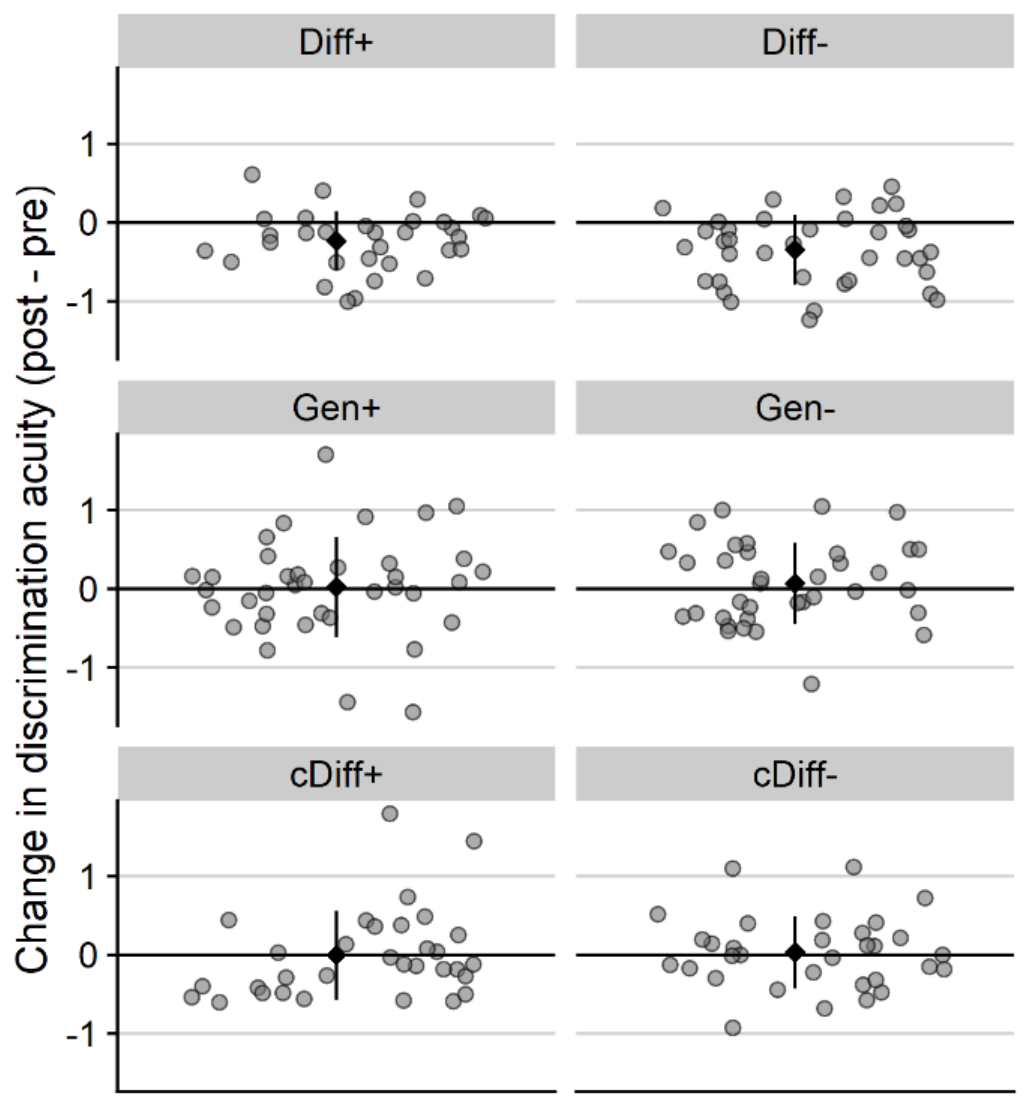

Figure 4: Individual change scores in discrimination acuity (post - pre standard deviation) per group. Positive/negative values indicate impaired/improved discrimination acuity, respectively. Black diamonds depict mean scores, while error bars represent aggregated standard deviations.

\section{CS-rating task}

US-expectancy ratings: Regarding US-expectancy, the 2 (learning type) x 2 (aversiveness) $\mathrm{x} 8$ (orientation) ANOVA revealed a marginal significant main effect of aversiveness, $F(1$, 128) $=3.70, p=.056, \eta_{p}^{2}=.03$ [CI: 0.00; 0.10], indicating slightly higher US-expectancy ratings for groups with aversive compared to neutral US. In addition, the main effect of learning type, $F(1,128)=25.87, p<.001, \eta_{p}^{2}=.17$ [CI: 0.07; 0.28], orientation, $F(5.61$, 718.43) $=37.90, p<.001, \eta_{p}{ }^{2}=.23$ [CI: 0.18; 0.27], and the interaction between orientation and learning type, $F(5.61,718.43)=3.69, p=.002, \eta_{p}^{2}=.03$ [CI: 0.01; 0.05], were significant. All groups showed the expected generalization gradient around the CS+. However, while differential learning groups demonstrated higher US-expectancy ratings for all orientations compared to the CS- orientation [CS+: $t(64)=8.89, p<.001, d=1.10 ; \mathrm{GS}+10^{\circ}$ : 
$t(64)=8.29, p<.001, d=1.03 ; \mathrm{GS}-10^{\circ}: t(64)=7.32, p<.001, d=0.91 ; \mathrm{GS}+20^{\circ}: t(64)=$ 5.25, $p<.001, d=0.65 ; \mathrm{GS}-20^{\circ}: t(64)=4.84, p<.001, d=0.60 ; \mathrm{GS}+30^{\circ}: t(64)=3.03, p=$ $.004, d=0.38$; GS-30 $: t(64)=2.70, p=.009, d=0.33$ ], generalization learning groups only revealed higher ratings for all orientations compared to the CS- except for the $+30^{\circ}$ and $30^{\circ}$ orientations [CS+: $t(66)=5.08, p<.001, d=0.62 ; \mathrm{GS}+10^{\circ}: t(66)=4.74, p<.001, d=$ 0.58 ; GS-10 $: t(66)=4.32, p<.001, d=0.53$; GS+20 $: t(66)=2.79, p=.007, d=0.34$; GS$20^{\circ}: t(66)=2.56, p=.013, d=0.31 ; \mathrm{GS}+30^{\circ}: t(66)=1.32, p=.191, d=0.16$; GS-30 $: t(66)$ $=0.55, p=.583, d=0.07$ ]. The 2 (target) $\times 2$ (aversiveness) 88 (orientation) ANOVA mainly confirmed these results, revealing significant main effects of aversiveness, $F(1,125)=$ 3.94, $p=.049, \eta_{p}^{2}=.03$ [CI: 0.00; 0.11], and orientation, $F(4.98,621.90)=48.58, p<.001$, $\eta_{p}{ }^{2}=.28$ [CI: 0.23; 0.32]. No effect involving the factor target was significant, $p s>.586$.

Threat ratings: Corresponding results for threat ratings can be found in the supplemental material.

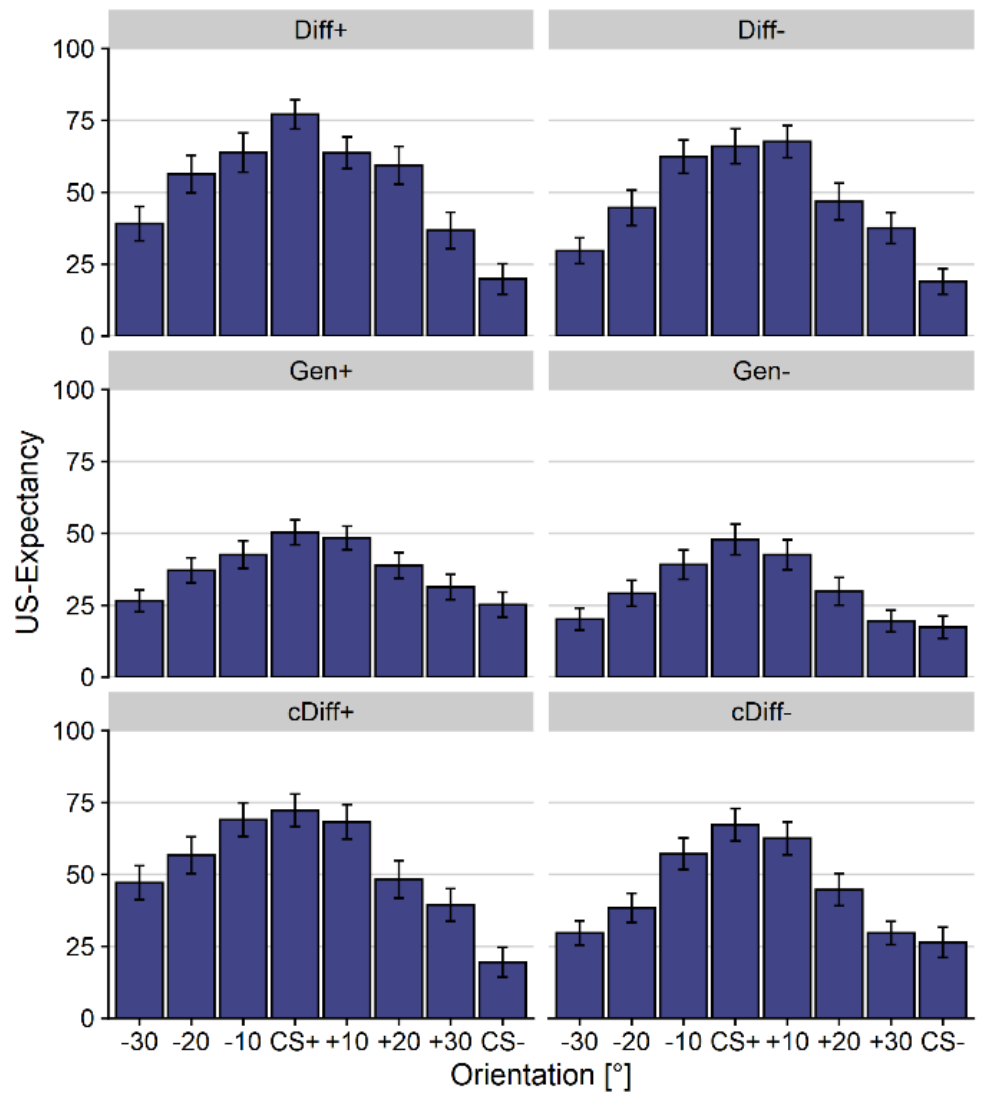

Figure 5: Mean US-expectancy ratings to the conditioned and generalization stimuli after associative learning. All groups demonstrated the expected response pattern for US- 
expectancy ratings. After associative learning, generalization learning groups showed generally lower US-expectancy ratings than differential learning groups. Error bars depict the standard error of the mean.

\section{Discussion}

The aims of this study were to establish a web-based aversive learning and discrimination task, which yields a continuous measure of discrimination acuity, and to test the hypothesis that aversive learning changes visual discrimination. To this end, we measured visual discrimination before and after differential associative learning, using grating stimuli varying in orientation. In addition, there were three types of controls. The first control conditions were presented with additional six grating stimuli during associative learning, similar to the study by McTeague et al. (2015). The second control conditions were psychophysically tested on the CS- instead of the CS+. And thirdly, for each of these groups, half of the participants were presented with neutral US, while the other half saw aversive US.

Accordingly, the US-validation task demonstrated successful picture stimulus manipulation. Groups that were confronted with aversive pictures rated the US as more arousing and more unpleasant than groups with neutral US, consistent with normative ratings of these pictures (Kurdi et al., 2017). Crucially, groups were able to memorize the US equally well, suggesting that participants paid adequate attention throughout the associative learning paradigm. Furthermore, threat and US-expectancy ratings revealed the expected generalization gradients after associative learning (McTeague et al., 2015; Struyf et al., 2015; Zaman et al., 2019). All groups expected the US more strongly after CS+ compared to CS- presentations, while expectancies decreased with increasing deviation from the CS+ orientation. Correspondingly, perceived threat was increased for participants in the groups seeing aversive US only (see supplemental material).

It has long been assumed that learning to predict threat prompts enhanced perceptual discrimination for threat-signaling stimuli (Miskovic \& Keil, 2012). However, recent studies show mixed results and enhanced (Rhodes et al., 2018) and impaired (Shalev et al., 2018) discrimination acuity after aversive learning have been reported. In our study, participants in the differential learning groups demonstrated narrower response distributions after associative learning, suggesting that associative learning sharpens 
visual discrimination, and thus, improves discrimination acuity. Contrary to expectations, this effect was not specific for aversive US, but could also be found in the differential learning group using neutral US. These results suggest that general stimulus salience, rather than threat-specific processes, prompt enhanced visual discrimination acuity. Pairing the CS with a complex, although neutral, picture stimulus, increases its salience since it is predicting a novel event (Kruschke, 2001; Mackintosh, 1975). This is especially true for between-group comparisons, where participants either see the aversive or the neutral stimuli. In addition, we cannot exclude that participants in the neutral condition did not expect aversive US in upcoming trials, as they were not told about viewing one category of US only. Consequently, the CS in the neutral condition might have gained motivational significance and thus salience. Crucially, motivationally significant stimuli receive prioritized attentional processing (Bradley, 2009; Sokolov, 1963).

On a neural level, it is well established that learning to detect threat-related signals is associated with enhanced activity in visuocortical areas (Miskovic \& Keil, 2012). In particular, it has been demonstrated that aversive learning induces neural orientation tuning in the primary visual cortex, leading to sparser neural representations of conditioned threat (Antov et al., 2020; McTeague et al., 2015). Yet, the mechanisms driving these changes are still under debate. It has been assumed that re-entrant projections from fearrelevant areas centered around the amygdala prompt short- and long-term adaptions in threat-related feature-specific neurons (Keil et al., 2009; Miskovic \& Keil, 2012). However, a recent study, exploiting the benefits of simultaneous fMRI-EEG analysis, showed that threat-related visuocortical changes were associated with neural activity in the ventral attention network, but not in the amygdala (Yin et al., 2020). The ventral attention network, including the temporoparietal and inferior frontal cortices, is specialized on detecting behaviorally relevant, salient stimuli (Corbetta \& Shulman, 2002). The results of our current study are well in line with the notion that the ventral attention network, activated by salient stimuli via associative learning, induces visuocortical tuning in orientation-specific neurons, which then leads to improved discrimination acuity in behavioral measures. Crucially, these changes do not depend on fear-relevant networks, which might be less activated by the neutral US used in the present study.

To support the hypothesis that stimulus predictiveness through associative learning leads to improved visual discrimination, we compared the experimental groups with control conditions, which were psychophysically tested on the unpaired stimulus (CS-) 
instead of the CS+. Crucially, the control conditions did not exhibit improved visual discrimination. Thus, enhanced discrimination acuity in the differential learning groups can neither be explained by training effects nor by sensitization, since the control groups were presented with the same US and the same amount of CS as the experimental group. These results are also in line with the findings of Rhodes et al. (2018), demonstrating reduced discrimination thresholds for the $\mathrm{CS}+$, but not for stimuli that were shifted by $90^{\circ}$ relative to the CS+.

The present study also included two further groups that did the same discrimination task as the differential learning groups but were presented with six additional generalization stimuli (GS) in $10^{\circ}$ steps around the CS+ $\left(-30^{\circ},-20^{\circ},-10^{\circ}, 10^{\circ}, 20^{\circ}, 30^{\circ}\right.$; see also McTeague et al., 2015) during the associative learning paradigm. These participants experienced directly that only the CS+ was followed by an US, while all other GS, even those very similar to the $\mathrm{CS}+$, signaled safety. Consequently, we assumed that the generalization learning groups would evidence even higher discrimination accuracies after learning since they were able to make a more discriminative learning experience compared to the differential learning groups. Yet, participants in the generalization learning groups did not exhibit changes in visual discrimination. On the contrary, analysis of US-expectancy even demonstrated generally reduced and a less distinct pattern of expectancy ratings to the CS and GS compared to the differential learning groups, indicating that including additional GS to the learning paradigm led to reduced, instead of improved discrimination. One simple explanation is that the generalization learning groups saw each stimulus only ten times compared to $40 \mathrm{CS}$ presentations in the differential learning groups. Here, our goal was to keep the total number of stimulus presentations equal in all groups. As a result, however, there were only ten US presentations as well, which could have led to reduced associative strength compared to differential learning groups, who saw 40 US (Kalish, 1954). In addition, there is evidence that changes in perceptual processing of threat occurs sometimes after extensive aversive learning only (Thigpen et al., 2017). On the other hand, it could be shown that visuocortical activity to conditioned threat increased after as few as two CS presentations (McTeague et al., 2015). It is important to mention, however, that the authors used a fully instructed aversive learning paradigm and that the extent to which these neural changes translate to behavioral measures of discrimination acuity still warrants further research. 
It is important to note that this study was conducted completely remotely, limiting controllability over external factors as the hard- and software used by the participant (e.g., display size), as well as environment variables (e.g., distractors), which are usually standardized in laboratory studies. However, this can also be considered as a strength of the current study since we found significant changes in visual discrimination despite these limitations. In addition, the US-validation and memory tasks confirmed that participants paid adequate attention to the US and perceived them according to their normative ratings. This is well in line with studies demonstrating that cognitive and affective processes can be validly studied with minimal costs using smartphone- or web-based paradigms (McGregor et al., 2021; Purves et al., 2019; Seow \& Hauser, 2021; Wise \& Dolan, 2020).

In conclusion, the present web-based study presents evidence that associative learning improves visual discrimination acuity. These changes are likely to be driven by enhanced stimulus salience through acquired predictiveness. However, future studies need to replicate these psychophysical findings and seek converging evidence from direct neurophysiological measures of electrocortical and functional imaging studies.

\section{References:}

Andreatta, M., Neueder, D., Herzog, K., Genheimer, H., Schiele, M. A., Deckert, J., Domschke, K., Reif, A., Wieser, M. J., \& Pauli, P. (2020). Generalization of Conditioned Contextual Anxiety and the Modulatory Effects of Anxiety Sensitivity. Neurotherapeutics. https://doi.org/10.1007/s13311-020-00831-8

Antov, M. I., Plog, E., Bierwirth, P., Keil, A., \& Stockhorst, U. (2020). Visuocortical tuning to a threat-related feature persists after extinction and consolidation of conditioned fear. Scientific Reports, 10(1), 3926. https://doi.org/10.1038/s41598-020-60597-z

Apergis-Schoute, A. M., Schiller, D., LeDoux, J. E., \& Phelps, E. A. (2014). Extinction resistant changes in the human auditory association cortex following threat learning. Neurobiology of Learning and Memory, 113, 109-114. https://doi.org/https://doi.org/10.1016/j.nlm.2014.01.016

Beck, A. T., Epstein, N., Brown, G., \& Steer, R. A. (1988). An inventory for measuring clinical anxiety: psychometric properties. Journal of consulting and clinical psychology, 56(6), 893.

Boylan, M. R., Kelly, M. N., Thigpen, N. N., \& Keil, A. (2019). Attention to a threat-related feature does not interfere with concurrent attentive feature selection. Psychophysiology, 0(0), e13332. https://doi.org/10.1111/psyp.13332

Bradley, M. M. (2009). Natural selective attention: orienting and emotion. Psychophysiology, 46(1), 1-11. https://doi.org/10.1111/j.14698986.2008.00702.x 
Buhr, K., \& Dugas, M. J. (2002). The Intolerance of Uncertainty Scale: psychometric properties of the English version. Behav Res Ther, 40(8), 931-945.

https://www.ncbi.nlm.nih.gov/pubmed/12186356

Corbetta, M., \& Shulman, G. L. (2002). Control of goal-directed and stimulus-driven attention in the brain. Nature Reviews Neuroscience, 3(3), 201-215. https://doi.org/10.1038/nrn755

Delignette-Muller, M. L., \& Dutang, C. (2015). fitdistrplus: An R Package for Fitting Distributions. Journal of Statistical Software; Vol 1, Issue 4 (2015). https://www.jstatsoft.org/v064/i04

http://dx.doi.org/10.18637/jss.v064.i04

Diamond, D. M., \& Weinberger, N. M. (1986). Classical conditioning rapidly induces specific changes in frequency receptive fields of single neurons in secondary and ventral ectosylvian auditory cortical fields. Brain Research, 372(2), 357-360. https://doi.org/https://doi.org/10.1016/0006-8993(86)91144-3

Friedl, W. M., \& Keil, A. (2019). Adaptive Changes in the Visuocortical Contrast Response to Spatial Frequency Stimuli: Dissociation Between Alpha-band Power and Driven Oscillations. J Vis, 19(10), 184-184. https://doi.org/10.1167/19.10.184

Friedl, W. M., \& Keil, A. (2020). Aversive Conditioning of Spatial Position Sharpens Neural Population-level Tuning in Visual Cortex and Selectively Reduces Alphaband Activity. bioRxiv, 2020.2011.2014.382960.

https://doi.org/10.1101/2020.11.14.382960

Gerlach, A., Andor, T., \& Patel, A. (2008). Die Bedeutung von Unsicherheitsintoleranz für die Generalisierte Angststörung Modellüberlegungen und Entwicklung einer deutschen Version der Unsicherheitsintoleranz-Skala. Zeitschrift für Klinische Psychologie und Psychotherapie, 37(3), 190-199. http://econtent.hogrefe.com/doi/abs/10.1026/1616-3443.37.3.190

Greenhouse, S., \& Geisser, S. (1959). On methods in the analysis of profile data. Psychometrika, 24(2), 95-112.

Heim, S., \& Keil, A. (2006). Effects of classical conditioning on identification and cortical processing of speech syllables. Exp Brain Res, 175(3), 411-424. https://doi.org/10.1007/s00221-006-0560-1

Holt, D. J., Boeke, E. A., Wolthusen, R. P. F., Nasr, S., Milad, M. R., \& Tootell, R. B. H. (2014). A parametric study of fear generalization to faces and non-face objects: relationship to discrimination thresholds [Original Research]. Front Hum Neurosci, 8(624). https://doi.org/10.3389/fnhum.2014.00624

Johnson, N. L., Kotz, S., \& Balakrishnan, N. (1995). Continuous univariate distributions, volume 2 (Vol. 289). John wiley \& sons.

Kalish, H. I. (1954). Strength of fear as a function of the number of acquisition and extinction trials. Journal of Experimental Psychology, 47(1), 1-9. https://doi.org/10.1037/h0053732

Kass, M. D., Rosenthal, M. C., Pottackal, J., \& McGann, J. P. (2013). Fear Learning Enhances Neural Responses to Threat-Predictive Sensory Stimuli. Science, 342(6164), 1389. https://doi.org/10.1126/science.1244916

Kastner, A. K., Pauli, P., \& Wieser, M. J. (2015). Sustained attention in context conditioning: Evidence from steady-state VEPs. Int J Psychophysiol, 98(3 Pt 2), 546-556. https://doi.org/10.1016/j.ijpsycho.2015.03.005

Keil, A., Sabatinelli, D., Ding, M., Lang, P. J., Ihssen, N., \& Heim, S. (2009). Re-entrant projections modulate visual cortex in affective perception: evidence from 
Granger causality analysis. Hum Brain Mapp, 30(2), 532-540.

https://doi.org/10.1002/hbm.20521

Kluge, C., Bauer, M., Leff, A. P., Heinze, H.-J., Dolan, R. J., \& Driver, J. (2011). Plasticity of human auditory-evoked fields induced by shock conditioning and contingency reversal. Proc Natl Acad Sci U S A, 108(30), 12545-12550.

https://doi.org/10.1073/pnas.1016124108

Kraus, N., \& Disterhoft, J. F. (1982). Response plasticity of single neurons in rabbit auditory association cortex during tone-signalled learning. Brain Research, 246(2), 205-215. https://doi.org/https://doi.org/10.1016/00068993(82)91168-4

Kruschke, J. K. (2001). Toward a Unified Model of Attention in Associative Learning. Journal of Mathematical Psychology, 45(6), 812-863. https://doi.org/https://doi.org/10.1006/imps.2000.1354

Kurdi, B., Lozano, S., \& Banaji, M. R. (2017). Introducing the Open Affective Standardized Image Set (OASIS). Behav Res Methods, 49(2), 457-470.

https://doi.org/10.3758/s13428-016-0715-3

Lang, P. J., Bradley, M. M., \& Cuthbert, B. N. (1997). Motivated attention: Affect, activation, and action. Attention and orienting: Sensory and motivational processes, 97, 135.

Li, W., Howard, J. D., Parrish, T. B., \& Gottfried, J. A. (2008). Aversive Learning Enhances Perceptual and Cortical Discrimination of Indiscriminable Odor Cues. Science, 319(5871), 1842. https://doi.org/10.1126/science.1152837

Lissek, S., Rabin, S., Heller, R. E., Lukenbaugh, D., Geraci, M., Pine, D. S., \& Grillon, C. (2010). Overgeneralization of conditioned fear as a pathogenic marker of panic disorder. Am J Psychiatry, 167(1), 47-55. https://doi.org/10.1176/appi.ajp.2009.09030410

Mackintosh, N. J. (1975). A theory of attention: Variations in the associability of stimuli with reinforcement [doi:10.1037/h0076778]. American Psychological Association.

McGregor, T., Purves, K. L., Constantinou, E., Baas, J. M. P., Barry, T. J., Carr, E., Craske, M. G., Lester, K. J., Palaiologou, E., Breen, G., Young, K. S., \& Eley, T. C. (2021). Largescale remote fear conditioning: Demonstration of associations with anxiety using the FLARe smartphone app. Depress Anxiety, $n / a(n / a)$.

https://doi.org/10.1002/da.23146

McTeague, L. M., Gruss, L. F., \& Keil, A. (2015). Aversive learning shapes neuronal orientation tuning in human visual cortex [Article]. Nat Commun, 6, 7823. https://doi.org/10.1038/ncomms8823

Miskovic, V., \& Keil, A. (2012). Acquired fears reflected in cortical sensory processing: a review of electrophysiological studies of human classical conditioning. Psychophysiology, 49(9), 1230-1241. https://doi.org/10.1111/j.14698986.2012.01398.x

Miskovic, V., \& Keil, A. (2013). Visuocortical changes during delay and trace aversive conditioning: evidence from steady-state visual evoked potentials. Emotion, 13(3), 554-561. https://doi.org/10.1037/a0031323

Picton, T. W., Bentin, S., Berg, P., Donchin, E., Hillyard, S. A., Johnson, R., Jr., Miller, G. A., Ritter, W., Ruchkin, D. S., Rugg, M. D., \& Taylor, M. J. (2000). Guidelines for using human event-related potentials to study cognition: recording standards and publication criteria. Psychophysiology, 37(2), 127-152. https://www.ncbi.nlm.nih.gov/pubmed/10731765 
Purves, K. L., Constantinou, E., McGregor, T., Lester, K. J., Barry, T. J., Treanor, M., Sun, M., Margraf, J., Craske, M. G., Breen, G., \& Eley, T. C. (2019). Validating the use of a smartphone app for remote administration of a fear conditioning paradigm. Behaviour Research and Therapy, 123, 103475.

https://doi.org/https://doi.org/10.1016/j.brat.2019.103475

Quirk, G. J., Armony, J. L., \& LeDoux, J. E. (1997). Fear Conditioning Enhances Different Temporal Components of Tone-Evoked Spike Trains in Auditory Cortex and Lateral Amygdala. Neuron, 19(3), 613-624.

https://doi.org/https://doi.org/10.1016/S0896-6273(00)80375-X

$\mathrm{R}$ Development Core Team. $R$ : A language and environment for statistical computing. In $\mathrm{R}$ Foundation for Statistical Computing.

Resnik, J., Sobel, N., \& Paz, R. (2011). Auditory aversive learning increases discrimination thresholds. Nature Neuroscience, 14(6), 791-796.

https://doi.org/10.1038/nn.2802

Rhodes, L. J., Ruiz, A., Ríos, M., Nguyen, T., \& Miskovic, V. (2018). Differential aversive learning enhances orientation discrimination. Cognition \& emotion, 32(4), 885891. https://doi.org/10.1080/02699931.2017.1347084

Seow, T. X. F., \& Hauser, T. (2021). Reliability of web-based affective auditory stimulus presentation. In: bioRxiv.

Shalev, L., Paz, R., \& Avidan, G. (2018). Visual Aversive Learning Compromises Sensory Discrimination. Journal of Neuroscience, 38(11), 2766-2779.

https://doi.org/10.1523/jneurosci.0889-17.2017

Sokolov, E. N. (1963). Perception and the conditioned reflex.

Stegmann, Y., Ahrens, L., Pauli, P., Keil, A., \& Wieser, M. J. (2020). Social aversive generalization learning sharpens the tuning of visuocortical neurons to facial identity cues. eLife, 9, e55204. https://doi.org/10.7554/eLife.55204

Stegmann, Y., Reicherts, P., Andreatta, M., Pauli, P., \& Wieser, M. J. (2019). The effect of trait anxiety on attentional mechanisms in combined context and cue conditioning and extinction learning. Scientific Reports, 9(1), 8855.

https://doi.org/10.1038/s41598-019-45239-3

Struyf, D., Zaman, J., Vervliet, B., \& Van Diest, I. (2015). Perceptual discrimination in fear generalization: Mechanistic and clinical implications. Neurosci Biobehav Rev, 59, 201-207. https://doi.org/10.1016/j.neubiorev.2015.11.004

Thigpen, N. N., Bartsch, F., \& Keil, A. (2017). The malleability of emotional perception: Short-term plasticity in retinotopic neurons accompanies the formation of perceptual biases to threat. Journal of Experimental Psychology: General, 146(4), 464-471. https://doi.org/10.1037/xge0000283

Wieser, M. J., Flaisch, T., \& Pauli, P. (2014). Raised middle-finger: electrocortical correlates of social conditioning with nonverbal affective gestures. PLOS ONE, 9(7), e102937. https://doi.org/10.1371/journal.pone.0102937

Wieser, M. J., Reicherts, P., Juravle, G., \& von Leupoldt, A. (2016). Attention mechanisms during predictable and unpredictable threat - A steady-state visual evoked potential approach. Neuroimage, 139, 167-175.

https://doi.org/10.1016/j.neuroimage.2016.06.026

Wise, T., \& Dolan, R. J. (2020). Associations between aversive learning processes and transdiagnostic psychiatric symptoms in a general population sample. Nature Communications, 11(1), 4179. https://doi.org/10.1038/s41467-020-17977-w

Yin, S., Bo, K., Liu, Y., Thigpen, N., Keil, A., \& Ding, M. (2020). Fear conditioning prompts sparser representations of conditioned threat in primary visual cortex. Social Cognitive and Affective Neuroscience. https://doi.org/10.1093/scan/nsaa122 
Zaman, J., Ceulemans, E., Hermans, D., \& Beckers, T. (2019). Direct and indirect effects of perception on generalization gradients. Behaviour Research and Therapy, 114, 4450. https://doi.org/https://doi.org/10.1016/j.brat.2019.01.006

\section{Supplemental material}

\section{Results}

\section{Threat ratings:}

After associative learning, the 2 (aversiveness) x 2 (learning type) x 8 (orientation) ANOVA yielded a significant two-way interaction between aversiveness and orientation, $F(5.04,600.15)=12.64, p<.001, \eta_{p}^{2}=.10$ [CI: 0.06; 0.13], while all effects involving learning type were not significant ( $p s>$.237). Before associative learning, no differences among groups and conditions could be found ( $p s>.120$ ). Similar results could be retrieved for the 2 (aversiveness) x 2 (target) x 8 (orientation) ANOVA after learning, revealing a significant interaction between aversiveness and orientation, $F(4.88,575.28)=17.90, p<$ $.001, \eta_{p}{ }^{2}=.13$ [CI: 0.09; 0.17], without any effect involving the factor target ( $p s>.276$ ). These results suggest that the groups that saw aversive US exhibited the expected generalization gradient around the CS+, while groups that saw neutral US did not (see Fig. 1S).

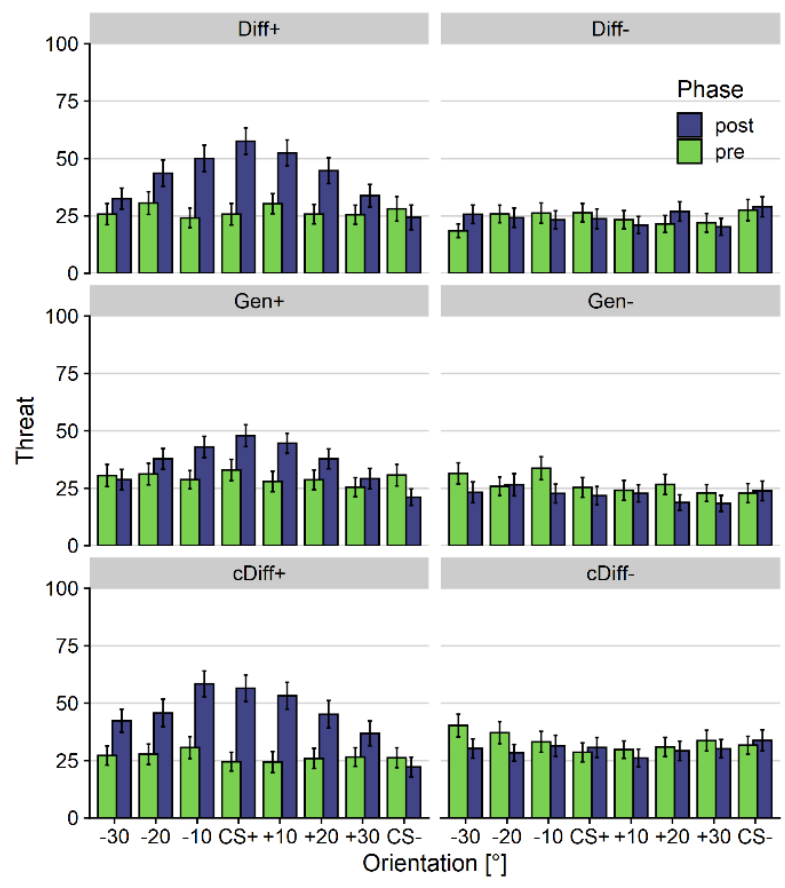

Figure 1S: Mean subjective threat ratings to the conditioned and generalization stimuli. Only groups with aversive US demonstrated the typical generalization gradient around the CS+ after associative learning. Error bars depict the standard error of the mean. 